Cahiers $d u$ MONDE RUSSE

\section{Cahiers du monde russe}

Russie - Empire russe - Union soviétique et États indépendants

$49 / 1 \mid 2008$

Réformes et société en Asie centrale tsariste et soviétique

\title{
Peindre et vivre en URSS dans les années 1920-1930
}

Commandes, engagements sous contrat et missions de création

\section{Cécile Pichon-Bonin}

\section{(2) OpenEdition}

\section{Journals}

Édition électronique

URL : https://journals.openedition.org/monderusse/9117

DOI : $10.4000 /$ monderusse. 9117

ISSN : $1777-5388$

Éditeur

Éditions de l'EHESS

Édition imprimée

Date de publication : 25 février 2008

Pagination : $47-74$

ISBN : 978-2-7132-2195-8

ISSN : $1252-6576$

Référence électronique

Cécile Pichon-Bonin, «Peindre et vivre en URSS dans les années 1920-1930», Cahiers du monde russe [En ligne], 49/1 | 2008, mis en ligne le 01 janvier 2009, consulté le 02 septembre 2022. URL http://journals.openedition.org/monderusse/9117; DOI : https://doi.org/10.4000/monderusse.9117 


\title{
CAIR N
}

chercher : repérer : avancer

Cet article est disponible en ligne à l'adresse :

http://www.cairn.info/article.php?ID REVUE=CMR\&ID NUMPUBLIE=CMR 491\&ID ARTICLE=CMR 4910047

\section{Peindre et vivre en URSS dans les années 1920-1930. Commandes, engagements sous contrat et missions de création par CÉcile PICHON-BONIN}

\author{
| Editions de l'EHESS | Cahiers du monde russe
}

2008/1 - Vol 49

ISSN 1252-6576 | ISBN 9782713221958 | pages 47 à 74

Pour citer cet article :

- Pichon-Bonin C., Peindre et vivre en URSS dans les années 1920-1930. Commandes, engagements sous contrat et missions de création, Cahiers du monde russe 2008/1, Vol 49, p. 47-74.

Distribution électronique Cairn pour les Editions de l'EHESS.

(C) Editions de l'EHESS. Tous droits réservés pour tous pays.

La reproduction ou représentation de cet article, notamment par photocopie, n'est autorisée que dans les limites des conditions générales d'utilisation du site ou, le cas échéant, des conditions générales de la licence souscrite par votre établissement. Toute autre reproduction ou représentation, en tout ou partie, sous quelque forme et de quelque manière que ce soit, est interdite sauf accord préalable et écrit de l'éditeur, en dehors des cas prévus par la législation en vigueur en France. Il est précisé que son stockage dans une base de données est également interdit. 
CÉCILE PICHON-BONIN

\title{
PEINDRE ET VIVRE EN URSS DANS LES ANNÉES 1920-1930
}

\author{
Commandes, engagements sous contrat \\ et missions de création
}

\begin{abstract}
À la mémoire de Ludmila Gaav
Les études sur l'art soviétique se répartissent selon trois axes principaux : une histoire esthétique ${ }^{1}$, une vision idéologisée largement inféodée à l'approche totalitarienne $^{2}$ et une histoire culturelle, plus encline à adopter les thèses « révisionnistes $»^{3}$
\end{abstract}

\begin{abstract}
1. Nous pensons notamment aux très nombreuses monographies et articles parus en URSS et en Russie, des années 1970 à nos jours, ou au texte d'Hubertus Gassner et Eckhart Guillen, « Ot sozdanija utopičeskogo porjadka $\mathrm{k}$ ideologii umorotvorenija $\mathrm{v}$ svete estetičeskoj dejstvitel'nosti », in Agitacija za sčastie, Sovetskoe iskusstvo stalinskoj epohi, catalogue de l'exposition du Musée russe de Saint-Pétersbourg et de la Dokumenta de Kassel, Brême : Temmen, 1994, p. 27-59.

2. Cette école de pensée hérite des travaux d'Hannah Arendt et de Raymond Aron sur le totalitarisme et postule un régime politique monolithique, un État tout-puissant exerçant un contrôle absolu sur une société endoctrinée, privée de droit, où règne la terreur. Les tenants de cette approche ont ainsi tendance à prendre l'ambition du régime pour la réalité et à négliger les tensions et les conflits pourtant bien réels. Dans le domaine de l'histoire de l'art, l'exemple le plus caractéristique en est sans doute l'ouvrage d'Igor Golomstock, L'art totalitaire : Union soviétique, III ${ }^{\mathrm{e}}$ Reich, Italie fasciste, Chine, P. : éditions Carré, 1991. D'autres études et catalogues d'exposition adoptent cependant, dans le même cadre de conception totalitarienne, des positions plus nuancées. Retenons en particulier : Matthew Cullerne Bown, Art under Stalin, Oxford : Phaidon, 1991 ; Aleksandr Morozov, Konec utopii : Iz istorii iskusstva v SSSR 1930h godov, M. : Galart, 1995 ; Vitalij Manin, Iskusstvo v Rezervacii, M. : Éditorial URSS, 1999.

3. Ce courant d'histoire sociale émerge dans les années 1970. Il prend le contre-pied des recherches antérieures en proposant une histoire «par le bas » et non « vue d'en haut». Des historiens, comme Stephen Cohen, Moshe Lewin ou Gábor Rittersporn, entendent dépolitiser le discours sur l'URSS, le «désidéologiser », s'interroger sur les rapports de la société avec ses institutions, rompre avec la conception démiurgique du Parti, cesser de privilégier le pouvoir politique comme objet principal d'analyse, au profit des sphères économique et sociale. Dans
\end{abstract}


et accordant généralement une place centrale à la littérature ${ }^{4}$. Bénéficiant aujourd'hui de l'ouverture des archives, notre démarche rejoint celle de récents travaux d'histoire politique et sociale ${ }^{5}$. Elle vise à « dépasser l'opposition entre l'interprétation révisionniste et la théorie totalitaire $»^{6}$ en proposant une histoire positiviste et en s'intéressant notamment aux conditions matérielles de la création picturale.

Lorsque des auteurs comme Igor Golomstock ou Vitalij Manin opposent la liberté de création des années 1920 à l'atmosphère répressive de la décennie suivante, ils associent ces phénomènes à deux contextes artistiques distincts. Sous cet angle, liberté rime avec marché de l'art et oppression avec commande d'État. Mais la lecture de la presse spécialisée de l'époque, des archives du MOSSH (Moskovskij Sojuz Hudožnikov - Union des artistes de Moscou) ${ }^{7}$, du Glavnauka (Glavnoe upravlenie po delam nauki i muzeev - Direction générale des institutions scientifiques et des musées ${ }^{8}$, de 1922 à 1933) puis du Glaviskusstvo (Glavnoe upravlenie po delam hudožestvennoj literatury i iskusstva - Direction générale des affaires littéraires et artistiques ${ }^{9}$, à partir de 1928), permet de découvrir un tableau moins binaire.

Tout d'abord, contrairement aux idées reçues, la commande (privée et publique) et le marché de l'art coexistent tout au long des années 1920 et 1930. Aussi la

cette perspective, les événements majeurs n'apparaissent plus comme le résultat d'une initiative politique émanant de Stalin, mais comme le produit de tensions internes. Deux principaux reproches ont été adressés à ce courant : des généralisations hâtives et une tendance à minimiser la terreur et la violence comme composantes importantes, voire majeures, du régime.

4. Nous renvoyons notamment aux travaux de Sheila Fitzpatrick : Cultural Revolution in Russia, 1928-1931, Bloomington-Londres : Indiana University Press, 1978 et The Cultural Front: Power and Culture in Revolutionary Russia, Ithaca-Londres : Cornell University Press, 1992.

5. Nous pensons en particulier aux récents travaux d'Alain Blum, de Nicolas Werth, d'Yves Cohen, aux thèses de doctorat d'Alexandre Sumpf («"Le visage vers la campagne". Les bolcheviks et l'éducation politique de la paysannerie dans les années 1920 », université Toulouse II - Le Mirail, 2006) et d'Élisabeth Essaïan («Le Plan général de la reconstruction de Moscou de 1935 : modèles, idées, acteurs », université Paris 8, 2006). En Russie, voir notamment Elena Osokina, Za fasadom «stalinskogo izobilija », M. : ROSSPEN, 1999 ou encore Galina Jankovskaja, Iskusstvo, den'gi i politika : hudožnik v gody pozdnego stalinizma, Perm : Gosudarstvennyj universitet, 2007. Ces auteurs ne se réclament ni d'un courant ni de l'autre, mais tentent de les concilier par l'histoire positiviste.

6. Alain Blum, Martine Mespoulet, L'anarchie bureaucratique : Statistique et pouvoir sous Staline, P. : La Découverte, 2003, p. 7.

7. L'Union est créée en 1932, après la dissolution des groupes artistiques. Contrairement à la littérature, aucune union n'encadre les arts plastiques à l'échelle russe et soviétique avant les années 1950. Le fonds d'archives est situé au RGALI (Rossijskij gosudarstvennyj arhiv literatury i iskusstva - Archives d'État de Russie de littérature et d'art), f. 2943.

8. Cette Direction générale dépend du Commissariat du peuple à l'Instruction publique (Narodnyj kommissariat prosveščenija - Narkompros ou NKP) dirigé par Anatolij Lunačarskij jusqu'en 1929. Son activité dans le domaine des arts plastiques est effective jusqu'en 1928, date à laquelle le Glaviskusstvo prend le relais. Son fonds d'archives se trouve au GARF (Gosudarstvennyj arhiv Rossijskoj Federacji - Archives d'État de la Fédération de Russie), f. A-2307.

9. Les fonctions de cette Direction (1928-1933) sont rapidement reprises par le département des arts plastiques du NKP. Le fonds est au RGALI, f. 645. 
pensée selon laquelle la période 1928-1932 constitue une rupture brutale doit-elle être nuancée ${ }^{10}$. Parallèlement aux achats et commandes privés, les possibilités de monnayer un tableau dans les années 1930 sont principalement de trois types. Premièrement, les «missions de création» (tvorčeskie komandirovki) perdurent. Mises en place dans les années 1920, elles permettent aux artistes de voyager pour les besoins de leur art en URSS comme à l'étranger. Deuxièmement, la pratique des commandes publiques se répand et, troisièmement, celle de l'engagement sous contrat (kontraktacija) se développe à partir de 1931. Distincte d'un contrat de commande, cette dernière pratique consiste à verser pendant un an un salaire mensuel à un artiste contre la livraison d'un nombre donné de tableaux. Nous nous proposons de reconstituer ici le fonctionnement de ces modes particuliers et de les situer par rapport à la sphère privée.

\section{Commandes privées et marché de l'art}

La période de la guerre civile est marquée par quelques commandes publiques dans les domaines de l'agitation et de la propagande ${ }^{11}$ (illustration, art monumental, décor de trains et de tramways, etc.). Peu rétribués, voire pas du tout, les peintres perçoivent plutôt des indemnités en nature ${ }^{12}$. Dans les années 1920, au moment de la NEP, les artistes obtiennent à nouveau le droit de s'associer et de constituer des groupes dans le but d'organiser des expositions et de vendre ainsi leurs œuvres. Le client privé demeure rare. Les principaux musées et l'État ${ }^{13}$ entrent en concurrence, chacun possédant sa propre commission d'achat et bénéficiant de budgets similaires. L'intervention de l'État n'est véritablement significative qu'à partir de 1927$1928^{14}$, quand la première commande d'envergure est passée à l'occasion des célébrations du dixième anniversaire de la révolution. Cette action est alors

10. Exceptée la thèse de Boris Groys postulant une continuité entre avant-garde et réalisme socialiste (B. Groys, Staline : CEuvre d'Art totale, Nîmes : éditions Jacqueline Chambon, 1990), l'idée prévaut d'une rupture au moment du Grand Tournant : ainsi Golomstock considère que la « méga-machine » totalitaire fut achevée à partir de 1932 (op. cit., p. 86). Cullerne Bown présente les années 1932-1933 comme un tournant irréversible et l'exposition de 1933 comme la fin d'une ère (op. cit., p. 35). Des études plus récentes situent la coupure au milieu des années 1930. (Voir Morozov, op. cit.,p. 54-55). Nous tenons également à remercier ici les participants au séminaire «Arts en transition », organisé par Valérie Pozner à l'ENS de 2004 à 2006, pour les fructueux échanges concernant la situation des différentes disciplines artistiques soviétiques dans les années 1928-1932.

11. À ce sujet, nous renvoyons à l'abondante iconographie et aux textes de l'ouvrage de V.P. Tolstoj, éd., Agitacionno-massovoe iskusstvo oformlenie prazdnestv, 1917-1932, M. : Iskusstvo, 1984

12. Dans ses mémoires, Ekaterina Zernova raconte que son groupe d'étudiants des Ateliers d'art et de technique (Vysšie hudožestvennye i tehničeskie masterskie, Vhutemas) a obtenu de la viande de cheval en échange de panneaux peints pour la caserne Krutickij. Voir E. Zernova, Vospominanija monumentalista, M. : Sovetskij hudožnik, 1985, p. 34.

13. À travers notamment le SNK (Sovet narodnyh komissarov - Conseil des commissaires du peuple), le RVS (Revoljucionnarnyj voennyj sovet - Conseil militaire révolutionnaire), ou le Glavnauka. 
accueillie positivement par les critiques ${ }^{15}$ et certains groupes d'artistes qui voient là une mesure égalitaire ${ }^{16}$ et un soutien apporté aux arts plastiques.

Au tournant des deux décennies, l'URSS connaît une crise profonde, et il faut attendre le milieu des années 1930 pour que la nouvelle intelligentsia stalinienne ${ }^{17}$ constitue une clientèle privée pour les œuvres d'art. Le retour en force de genres comme la nature morte, le portrait et le paysage, témoigne de ce phénomène, tout autant que certains types d'expositions. Ainsi, à partir de 1931, le Vsekohudožnik (Vserossijskij kooperativ hudožnikov - Coopérative des artistes de Russie) organise une exposition bazar (ill. 1). Il y revend aux acheteurs privés et aux organismes publics des tableaux qu'il a parfois acquis préalablement auprès des artistes. Mais le Vsekohudožnik joue aussi le rôle d'une galerie : il expose les œuvres des peintres et prélève un pourcentage sur la vente ${ }^{18}$. Citons également les expositions saisonnières rétablies en 1935 qui présentent des tableaux créés hors commande et des invendus résultant de commandes diverses, dressant ainsi un état des lieux de la création soviétique ${ }^{19}$.

Les clients individuels, donc les commandes privées, n'ont jamais cessé d'exister, mais la difficulté d'accès à une documentation suffisante obère ici une étude approfondie. Nous ne pouvons constater ce fait qu'à travers quelques exemples. Dans les années 1920, certains membres de la très conservatrice Association des artistes de la Russie révolutionnaire cultivent des liens privilégiés avec des dirigeants, comme Kliment Vorošilov, dont ils réalisent des portraits. Ces contacts se poursuivent dans la décennie suivante. En 1936, David Sterenberg témoigne de l'émergence d'une nouvelle clientèle et de la pratique de la commande privée. Il

14. Et ce malgré l'existence, depuis 1925, d'une commission chargée des commandes et des achats d'œuvres d'art au sein du Glavnauka.

15. Au moment de la première grande commande d'État en 1928, la presse fait état d'une conjoncture difficile pour les artistes due à la rareté des clients. Voir I. Hvojnik, « Izobrazitel'noe iskusstvo : Itogi i perspektivy » [Les arts plastiques : Bilans et perspectives], Sovetskoe iskusstvo, ${ }^{\circ} 5,1927$, p. 58-70; Id., «Jubilejnye vystavki : vystavka gosudarstvennyh zakazov k 10-letiju Oktjabrja » [Les expositions commémoratives : l'exposition de la commande d'État pour les dix ans d'Octobre], Sovetskoe iskusstvo, n 1, 1928, p. 20-35.

16. Voir, par exemple, l'argumentaire développé par l'OST (Ob̌̌čestvo hudožnikovstankovistov - Société des artistes de chevalet) et 4 iskusstva [les Quatre Arts] dans le débat qui les oppose à l'AHRR (Assocjacija hudožnikov revoljucionnarnoj Rossii - Association des artistes de la Russie révolutionnaire). En 1928, l'AHRR deviendra l'AHR (Assocjacija hudožnikov revoljucii - Association des artistes de la révolution).

17. À partir du milieu des années 1930, le terme d'intelligentsia ne désigne plus seulement les artistes et intellectuels mais aussi l'ensemble de la nouvelle élite sociale. L'emploi de ce vocable souligne le niveau culturel que cette classe privilégiée est censée avoir atteint.

18. Sur les ventes comme sur les commandes, le bureau central du MOSSH et le Vsekohudožnik prélèvent un pourcentage (de 12 à $20 \%$ ). Toute transaction doit normalement s'effectuer avec l'accord du secteur pictural du MOSSH, mais, au moins jusqu'au milieu des années 1930 , les artistes contournent fréquemment cette obligation, sans se soustraire pour autant aux contraintes pécuniaires.

19. Voir D. Denisov, «Vesennjaja vystavka moskovskih zivopiscev», Tvorčestvo, no 8 , 1935 ; «Vesennjaja vystavka», Sovetskoe iskusstvo, n² 23, 17 mai 1935 ; «Moskovskaja osenjaja vystavka », Sovetskoe iskusstvo, 5 juin $1935, \mathrm{n}^{\circ} 25$ 

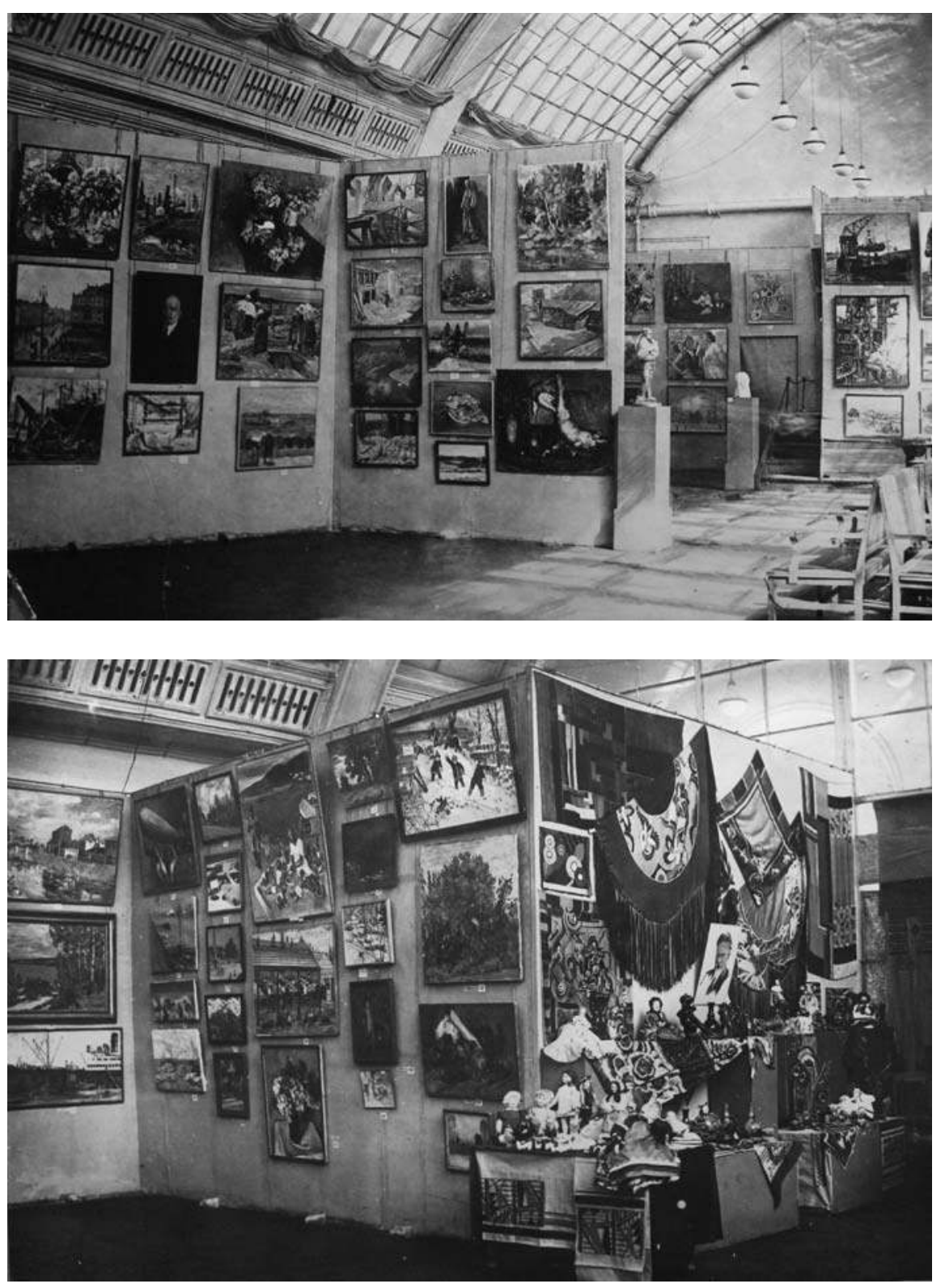

Illustration 1. Exposition-bazar organisée par le Vsekohudožnik, en 1931. Archives de la bibliothèque du MOSSH. 
raconte ainsi comment il a travaillé à un portrait du général Primakov ${ }^{20}$ posant dans une robe de chambre rapportée de Chine ${ }^{21}$.

Il n'est certes pas question de remettre en cause l'instance de la thématique soviétique, notamment stalinienne, dans la peinture, mais comment ne pas rappeler ces nombreux tableaux largement présents dans les expositions, aux sujets les plus divers : nature morte, paysage, nu, portrait non officiel. En outre, ces œuvres ne sont pas uniquement des produits d'atelier. Le flou des directives, le désordre ambiant, les difficultés de concertation des commanditaires (État, Parti, musées, organisations socioprofessionnelles), la participation active des peintres tant à la conception qu'à la sélection et aux jurys des expositions, tous ces éléments - qui font la spécificité du système soviétique - constituent de réelles contraintes pour les artistes, mais leur permettent aussi d'influer sur le choix et le traitement de leur sujet, et ce jusqu'au milieu des années 1930.

\section{Commande publique, commande d'État}

Une première idée à remettre en cause est celle d'une équivalence entre commande publique et une commande d'État qui serait directive, omniprésente et exclusive. Plusieurs commanditaires peuvent agir, voire interagir. Les commandes proviennent ainsi des grandes administrations : SNK, RVS, NKP et VCSPS (Vsesojuznyj central'nyj sovet professional'nyh sojuzov - Conseil central des syndicats d'URSS) principalement, des organisations socioprofessionnelles (MOSSH et Vsekohudožnik), des éditions d'art d'État (Gosudarstvennoe izdatel'tsvo izobrazitel'nyh iskusstv - Izogiz), des musées (essentiellement la galerie Tret'jakov et le musée de la Révolution), du Mossovet (Moskovskij sovet - Conseil de Moscou), mais aussi des industries ou des kolkhozes.

Bien entendu, ces acteurs n'interviennent ni dans les mêmes proportions ni avec la même fréquence ou régularité, leurs moyens sont différents, et leurs demandes conditionnent diversement la réalisation des œuvres. Cependant, trois modes de commande se détachent : le contrat ponctuel, le concours et la commande institutionnelle commémorative.

\section{2-1. Les trois principaux modes de commande publique}

Le premier type de commande prend la forme d'une collaboration ponctuelle entre un artiste ou un groupe d'artistes (appelé «brigade ») et un organisme. Le ou les tableaux sont alors conçus en fonction d'un lieu précis d'accrochage. Le

20. Evgenij Primakov, général de l'Armée rouge, est alors le nouveau compagnon de Lili Brik. Il comptera parmi les victimes des purges.

21. RGALI, f. 2943, op. 1, d. 69, Stenogramma obščego sobranija MOSSHa o bor'be za iskusstvo socjalističeskogo realizma protiv formalizma, 5.IV.1936,1. 54 . 
commanditaire sollicite le peintre directement, soit parce qu'il le connait, soit parce que l'artiste travaille sur le thème souhaité22. Le client peut aussi s'adresser au MOSSH ou au Vsekohudožnik et, lors d'une réunion, les artistes définissent plus précisément le programme iconographique de l'œuvre et déterminent la liste des créateurs qui s'en chargeront. Industries, kolkhozes, clubs, cantines... sont les utilisateurs privilégiés de ces commandes ponctuelles qui mobilisent peu de peintres et exigent des moyens relativement faibles. Il s'agit pour l'essentiel de commandes de fresques ou de panneaux décoratifs.

Le deuxième mode de commande, le concours, n'apparaît qu'au début des années 1930. Ainsi en 1931, la galerie Tret'jakov annonce dans le journal Sovetskoe iskusstvo ${ }^{23}$ qu'elle prépare une exposition et invite les peintres à présenter leurs esquisses. L'encart stipule que les travaux sélectionnés par le jury, composé de représentants des organisations socioprofessionnelles et des institutions, feront ensuite l'objet d'une commande. Ce mode est souvent utilisé par les musées ou les éditions d'État lors de l'organisation d'un événement particulier (exposition, fête, publication...). Ajoutons que le MOSSH, qui coorganise des expositions avec le Vsekohudožnik, opte parfois pour un système de prix et de récompenses visant à remplacer ou à accompagner certaines commandes ${ }^{24}$. Les récompenses s'imposent à partir de la fin des années 1930, lorsque le tri de la production artistique s'accentue. Les prix Stalin, mis en place en 1941, en sont certainement l'exemple le plus connu.

Quant à la commande d'État, supervisée - mais non complètement organisée par les principaux commissariats (équivalents des ministères), elle s'adresse simultanément à un nombre important d'artistes. Les commandes interviennent pour de grandes occasions, généralement au moment des fêtes anniversaires de la révolution d'Octobre, soit théoriquement en 1927, 1932 et 193725. Cette périodicité et la

22. Aleksandr Labas est sollicité pour un panneau car il travaille sur le thème de l'aviation.

23. «Vsem sovetskim hudožnikam » [À tous les artistes soviétiques], Sovetskoe iskusstvo, 3 juillet 1931

24. Voir RGALI f. 2943, op. 1, d. 67, Stenogramma obščego sobranija MOSSHa po obsuždeniju vystavki « 40 hudožnikov », 28.II.1936, 1. 4. Le document mentionne quatre niveaux de récompenses : 10 000, 7 000, 5000 et 3000 roubles, dans le cadre de l'exposition des femmes artistes du 8 mars 1934. Il est aussi question de récompenser les meilleures œuvres issues des missions ou des engagements sous contrat (voir Ju. Slavinskij, « Novaja kontraktacija », Bjulleten' Vsekohudožnika, n 1-2, 1935, p. 12), mais nous n'avons pas trouvé trace de concrétisation de ces intentions.

25. En 1928, le public peut visiter l'exposition célébrant les dix ans de l'Armée rouge, financée surtout par le RVS, et celle commémorant les dix ans d'Octobre, soutenue par le NKP et le SNK. L'organisation de cette dernière est bien lancée en 1927, mais les lenteurs administratives et les soucis matériels ont reporté son ouverture à l'année suivante. Par la suite, nous retrouvons ce mode d'intervention, qui consiste à associer aux fêtes révolutionnaires une grande exposition et une vaste commande passée aux artistes. En 1932, le schéma d'une double manifestation est identique. SNK, NKP et Vsekohudožnik allouent chacun 100000 roubles pour réaliser l'exposition «Quinze ans d'art soviétique », l'Izogiz et le MOSSH la subventionnent également. En 1935, la presse diffuse des informations relatives à la préparation d'une grande exposition pour commémorer les vingt ans d'Octobre deux ans plus tard avec pour thème «l'industrie du socialisme». Mais la mort en 1937 d'Ordžonikidze, commissaire du peuple à l'industrie lourde, principal commanditaire de l'exposition, et les difficultés d'organisation 
modicité des rétributions ne permettent pas à la vie artistique de reposer entièrement sur ces commandes d'État. Cependant, le caractère majeur, bien que ponctuel, de ces événements invite à détailler le processus.

\section{Les commandes institutionnelles à l'occasion de la commémoration de la révolution d'Octobre}

L'exemple des commandes anniversaires se révèle particulièrement surprenant. Tout d'abord, les seules productions d'Aleksandr Dejneka, Jurij Pimenov, Aleksandr Labas, Aleksandr Tyšler ou David Sterenberg26 (ill. 2) prouvent assez que la commande ne fut pas synonyme d'étouffement de la création et n'a pas empêché la réalisation d'œuvres de qualité. Comment se font effectivement les commandes? Qui sont les principaux décideurs? Quels sont les termes des contrats?

Les arts plastiques n'occupent qu'une place relativement marginale au sein des institutions étatiques, loin derrière la littérature, le théâtre ou le cinéma. Cela se reflète au niveau du déroulement même des commandes. Le plus souvent, ce sont des artistes, des groupes puis des organisations socioprofessionnelles qui sont à l'origine d'une exposition, et même des commandes. C'est notamment le cas en 1927 lorsque l'AHRR propose d'organiser une exposition avec le RVS, pour célébrer le quinzième anniversaire de l'Armée rouge ${ }^{27}$. On doit se défier de la tendance croissante de la presse de l'époque à accréditer l'idée que l'initiative d'expositions et de commandes provient d'un "génial instigateur », extérieur à la sphère artistique. Dans les années 1930, ce sont généralement les organisations socioprofessionnelles (Vsekohudožnik et MOSSH) qui prennent les initiatives. En 1932, la proposition d'une exposition célébrant les quinze ans du régime émane d'une commission spéciale présidée par A.S. Bubnov ${ }^{28}$, successeur d'Anatolij Lunačarskij à la tête du NKP. Le SNK entérine le projet. Dans un premier temps, il n'est pas envisagé de passer de commandes aux artistes, mais de puiser dans les réserves des musées et autres organismes. Les commandes interviendront par la suite à la demande des

courantes ont considérablement retardé la mise en place de l'événement. De fait, l'exposition ne sera montée qu'en 1939 et est restée célèbre comme exposition thématique et non en tant qu'exposition anniversaire.

26. Ces artistes sont tous membres de l'OST, groupe d'artistes des années 1920 proposant une figuration nourrie des réflexions des avant-gardes.

27. Cet événement n'a rien de surprenant. Depuis sa création, l'AHRR se révèle très dynamique et sollicite les administrations pour obtenir commandes et subventions.

28. RGALI f. 643, Vystavka «Hudožniki RSFSR za 15 let », op. 1, d. 2, Perepiska komissij vystavki, 7.IV.1932-5.IV.1933. Kopii protokola $n^{\circ} 16$ zasedanija SNK ot 7.VII.1932. La commission est composée d'A.S. Bubnov, Jakovlev, Slavinskij, Sulikov, Komarov. En 1932, le SNK charge ces personnes de la préparation de dioramas et de nouveaux pavillons pour les quinze ans d'Octobre. La proposition d'une exposition anniversaire, destinée à montrer l'évolution de la peinture soviétique durant les quinze années qui ont précédé, émane de cette commission. Le SNK entérine la proposition et débloque 100000 roubles, prélevés sur son fonds de réserve. La commission se voit chargée de l'organisation effective. 


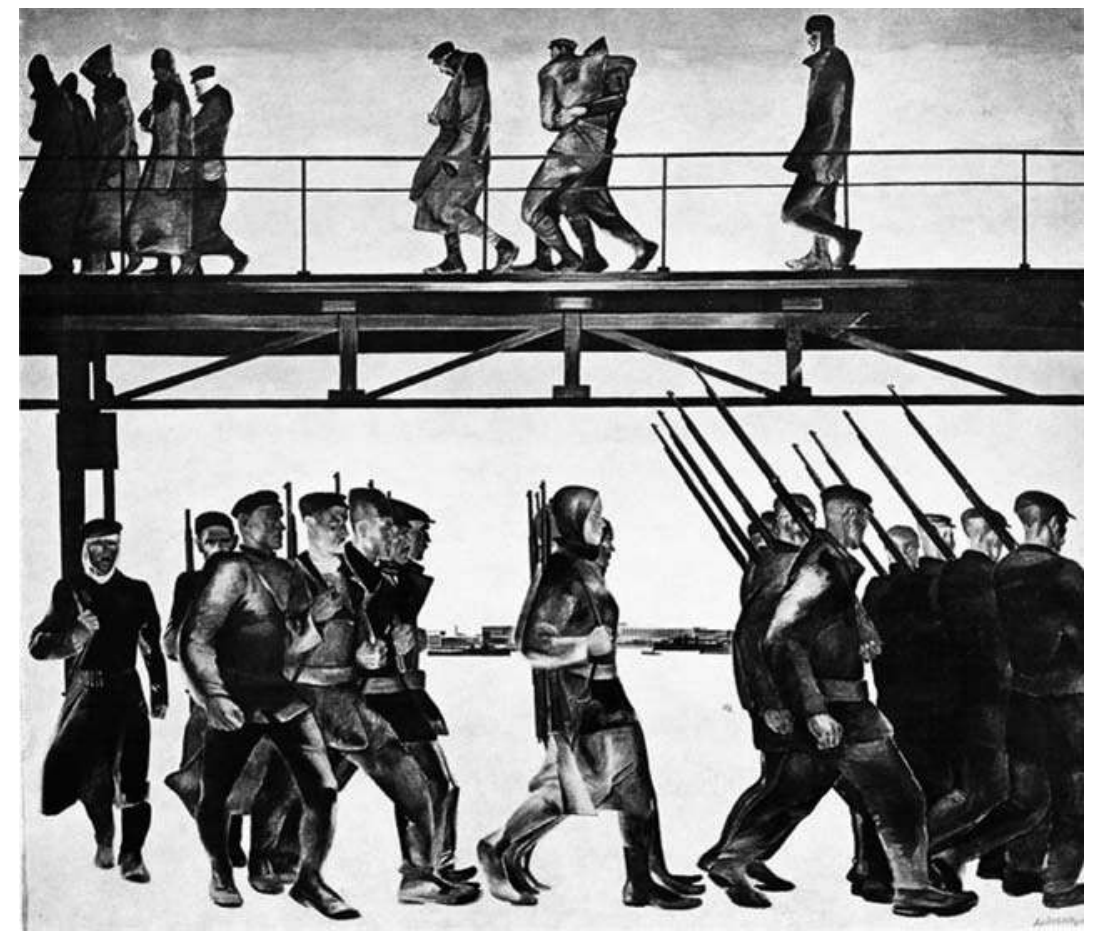

Illustration 2a. Aleksandr Dejneka, La défense de Petrograd, 1964 (copie de l'œuvre réalisée en 1927), huile sur toile, $210 \times 238 \mathrm{~cm}$, Galerie Tretjakov, Moscou, dr.

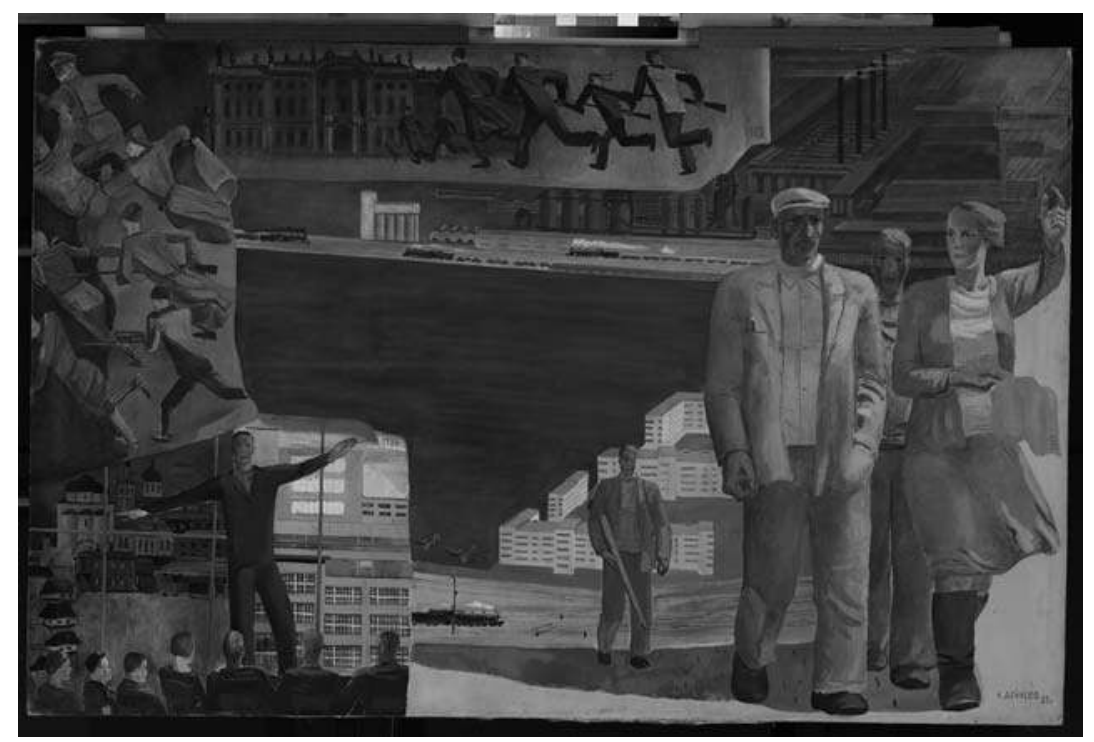

Illustration 2b. Aleksandr Dejneka, Qui l'Emportera ?, 1932, huile sur toile, $129,5 \times 200 \mathrm{~cm}$, Galerie Tretjakov, Moscou, dr. 


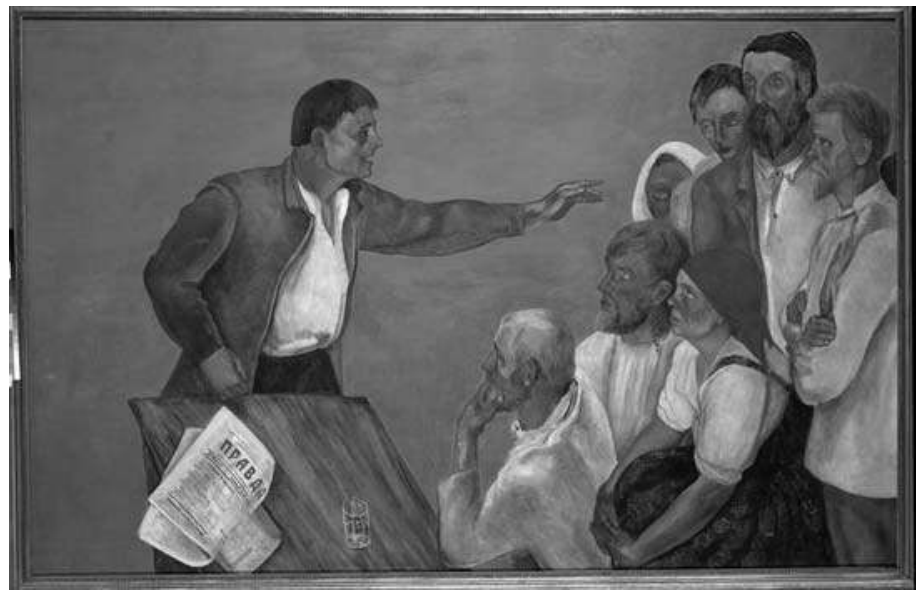

Illustration 2c. David Sterenberg, L'agitateur, 1927, huile sur toile, $135 \times 208 \mathrm{~cm}$, Galerie Tretjakov, Moscou, dr.

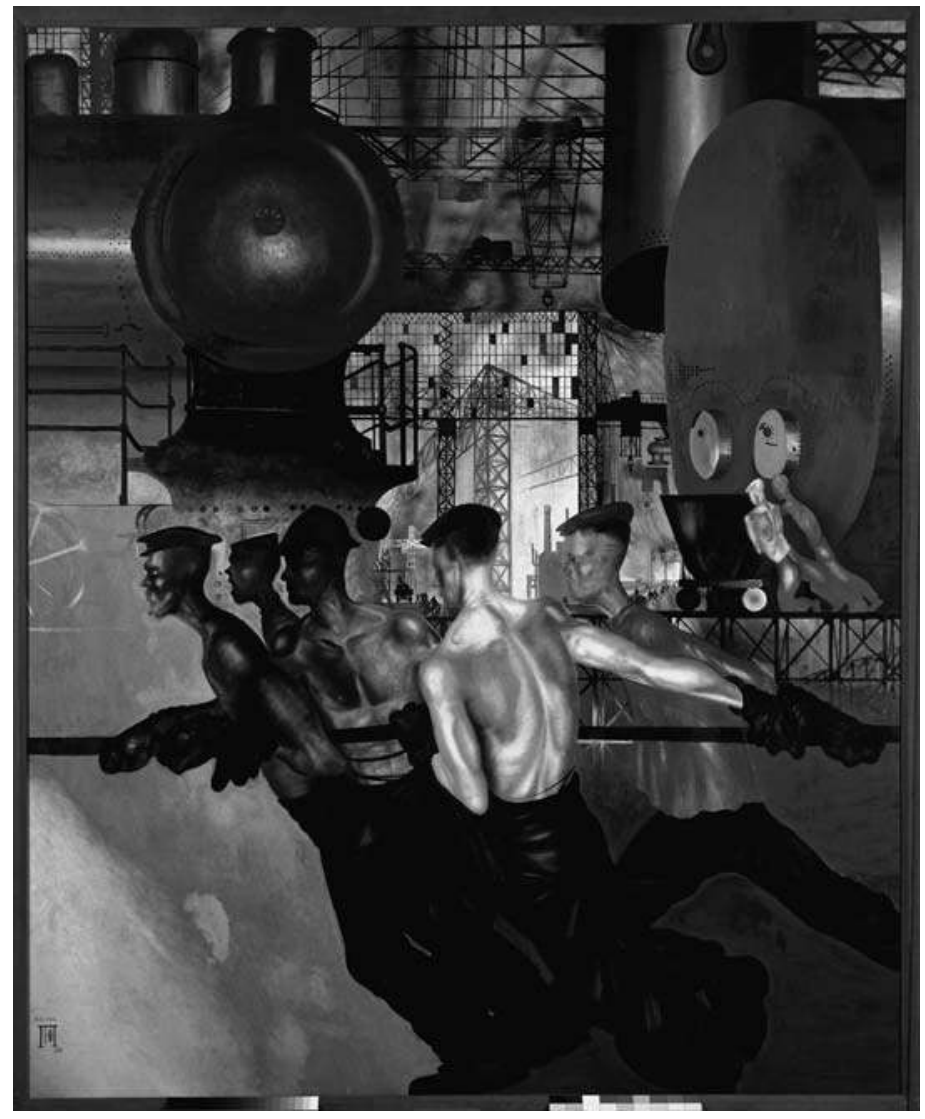

Illustration 2d. Jurij Pimenov, Crée l'industrie lourde !, 1927, huile sur toile, $260 \times 212 \mathrm{~cm}$, Galerie Tretjakov, Moscou, dr. 
organisations socioprofessionnelles, dans des conditions suffisamment originales pour que l'on s'y attarde.

\section{"Quinze ans d'art soviétique » : une commande à rebours}

Prétextant le manque d'œuvres relatives à certaines thématiques, les peintres responsables du secteur pictural du MOSSH demandent à A.S. Bubnov d'allouer une partie du budget aux commandes ${ }^{29}$. Finalement, la majorité des œuvres exposées proviendra de ce dispositif, non prévu par les dirigeants ${ }^{30}$. Dans ces conditions, la commande anniversaire de 1932 étonne et ne correspond pas à l'idée que l'on pourrait se faire d'une commande institutionnelle dans l'URSS du début des années 1930. En effet, le comité de direction de l'exposition commence par lancer une vaste enquête auprès des artistes ${ }^{31}$ : ceux-ci sont invités à signaler les œuvres achevées disponibles et celles en cours de réalisation susceptibles de l'être pour le 30 septembre qui, si elles sont en accord avec le plan thématique, pourront être retenues pour une commande ${ }^{32}$.

Cette démarche rompt totalement avec le principe même de la commande artistique, qui implique un programme défini au préalable. En outre, le terme même de commande recouvre ici une réalité différente, puisque le commanditaire ne devient pas nécessairement propriétaire de l'œuvre. Ce système combine vente de tableaux et aide financière à la création. Les jurys, composés des peintres eux-mêmes, examinent les propositions. Ils peuvent décider d'attribuer une somme d'argent en vue de l'achèvement d'un tableau donné, selon l'état de son avancement, son degré de difficulté et la qualification de l'artiste. Le secrétariat du MOSSH fixe ainsi deux niveaux d'aide, 500 et 700 roubles, ce qui correspond à un prix de vente assez bas pour un tableau achevé et équivaut au salaire mensuel d'une personne occupant l'importante fonction de commissaire du peuple ${ }^{33}$. L'âge et la notoriété de l'artiste constituent des critères fondamentaux pour arrêter ce classement. Les sommes versées représentent en principe une avance sur la vente à venir, leur attribution faisant l'objet d'un contrat spécifique. Si le tableau ne trouve pas acquéreur, le

29. Archives de la galerie Tret’ jakov, 8 II/482, 1. 45.

30. A. Morozov, «K istorii vystavki 'Hudožniki RSFSR za 15 let' (Leningrad-Moskva, 1932$1935) »$ [L'histoire de l'exposition « Quinze ans d'art soviétique » (Leningrad-Moscou, 19321935)], Sovetskoe iskusstvoznanie, vol. 1 (16), M.: 1983, p. 123. L'auteur indique que la somme totale pour cette commande s'élève à 150000 roubles, ce qui dépasse de deux tiers les assignations habituelles du NKP pour les arts plastiques à cette période.

31. RGALI f. 643, op. 2, d. 1. Protokoly zasedanij členov žjuri, 17.VIII. 1932.

32. Morozov, art. cit., p. 123.

33. Voir Vladimir Paperny, Kul'tura dva, [1985], M.: 1996, p. 123. Selon S.Žuravlev, M. Muhin, «Krepost' socializma » : Povsednevnost' i motivacija truda na sovetskom predprijatii, 1928-1938 gg., M. : ROSSPEN, 2004, p. 59, en 1932 le salaire mensuel moyen d'un ouvrier de la capitale s'élève à 158 roubles. 
comité d'exposition statue au cas par cas pour demander le remboursement. De fait, il est peu probable que l'Union recouvre ses fonds.

Le Vsekohudožnik investit des sommes plus importantes, pour une contrepartie différente. Un peintre peut recevoir de 700 à 1200 roubles d'aide, au prorata des dimensions de la toile. Dans les faits, les décideurs intègrent d'autres paramètres ${ }^{34}$. Le peintre bénéficiaire cède la propriété de l'œuvre ainsi que les droits de reproduction et d'exposition au Vsekohudožnik ${ }^{35}$. Celui-ci peut ensuite revendre les tableaux. Il est permis d'imaginer un principe de spéculation. L'opération de soutien à la création prend ici étrangement la forme d'une commande a posteriori. C'est dans ces conditions que Dejneka termine son célèbre tableau Qui l'emportera ?Les organisations socioprofessionnelles, et par là même les artistes, jouent donc un rôle central. Commanditaires elles-mêmes, elles sont aussi des intermédiaires obligés et les organisateurs principaux des commandes et expositions.

Après avoir souligné les conditions particulières de la commande à l'occasion du quinzième anniversaire de la révolution, il convient de s'arrêter sur un deuxième élément, étroitement lié à la commande artistique : l'accord préalable. Quelles données contractuelles unissent les parties? Quels sont les délais, les prix et les conditions techniques de réalisation? Comment le programme iconographique est-il défini ?

\section{2-2. Les modalités des contrats}

Les contrats étudiés ici ont été établis entre le RVS et Dejneka d'une part, et P. Williams d'autre part, dans le cadre de l'exposition commémorant les dix ans de l'Armée rouge ${ }^{36}$. Ils sont relatifs aux esquisses des tableaux La défense de Petrograd et Manifestation dans le Sud en 1919, et datent de février et mars 1927. Par ailleurs, nous avons retenu un contrat de travail passé en février 1934 entre la VOKS (Vsesojuznoe ob̌̌čestvo kul turnoj svjazi s zagranicej - Association soviétique pour les relations culturelles avec l'étranger) et une brigade de peintres composée de S. Adlivankin, V. Liušin, S. Antonov, A. Niskij et A. Dejneka ${ }^{37}$. L'examen de ces documents fait apparaitre un profil type de contrat.

\footnotetext{
34. David Sterenberg obtient en effet 1200 roubles contre 1000 pour Aleksandr Gerasimov, alors que ce dernier travaille à un tableau plus grand. Quels sont alors les critères décisifs ? La cote de l'artiste ? L'avancement du travail ? Ou simplement le fait que Sterenberg préside la séance alors que Gerasimov est absent ?

35. RGALI f. 643, op. 2, d. 1, Protokol zasedanij žjuri ot 10.VIII.1932.

36. RGALI f. 2941, AHR, op. 1, d. 53, Prezidium dogovora s hudožnikami (bukvy A-L) na vypolnenie rabot s $\mathrm{X}$ vystavki AHRR.

37. GARF f. 5283, VOKS, op. 11, Otdel vystavok, d. 302,1. 13.
} 


\section{Constantes et variantes}

Dans tous les cas, le contrat stipule le sujet de l'œuvre, les délais de réalisation, la technique (huile sur toile) ; il comporte des indications relatives aux dimensions du support, la rétribution du peintre et les modalités de son versement. Enfin, il peut être demandé à l'auteur de fournir le cadre avec l'œuvre. À ces conditions générales, communes aux types de contrat de commandes depuis la Renaissance, s'ajoutent certaines modalités.

Dans le souci d'un contrôle et d'un suivi rigoureux du travail, les esquisses font l'objet d'une attention particulière, voire d'un contrat spécifique. En 1927, selon l'accord établi avec le RVS, Dejneka perçoit 500 roubles $^{38}$ en trois versements : le premier à la signature (200 roubles), le deuxième lors de l'examen par le comité de la première esquisse (100 roubles) et le dernier lors de la remise de l'esquisse finale (200 roubles). À chaque étape, le contrat peut être rompu si le travail ne convient pas ou si l'artiste ne tient pas compte des remarques du comité chargé d'évaluer les esquisses aux différents stades de l'élaboration ${ }^{39}$. L'œuvre fait ensuite l'objet d'un second accord. En 1934, un contrat unique entre la VOKS et la brigade conduite par Adlivankin concerne à la fois l'esquisse et l'œuvre achevée. Mais la procédure reste la même ${ }^{40}$.

Dans les contrats conclus en 1927 avec le RVS figure une clause relative aux droits d'auteur. Dejneka conserve ainsi un droit moral sur ses esquisses qui deviennent la propriété du RVS. Le peintre cède un droit partiel sur d'éventuelles reproductions et expositions effectuées pour les besoins du RVS, mais s'il a l'occasion de reproduire et de diffuser ses œuvres par un autre biais, tel l'Izogiz, il sera rétribué selon les tarifs en vigueur. Le RVS bénéficie d'un droit d'exploitation non exclusif ; cependant, aucune copie sur toile ne peut être exécutée sans son accord.

Un dernier aspect concerne le soutien apporté aux artistes dans la mise en œuvre et la réalisation de leur projet. En 1927, l'artiste s'engage à se documenter sur le thème retenu et à consulter textes littéraires, documents, illustrations et tout matériau y afférents. Ce souci de «fidélité à la vérité historique », mis en avant dans le contrat, reste du ressort du peintre. Dans les années 1930, les commanditaires ont tendance à s'emparer davantage de cet aspect. Ainsi, en 1932, le RVS organise des séminaires à l'intention des artistes en prévision de l'exposition commémorative des quinze ans de l'Armée rouge. Ces conférences abordent différents thèmes

38. Nous ne disposons pas de données pour les années 1927-1928, mais en 1929, le salaire moyen d'un ouvrier s'élevait à 93 roubles. Voir S. Žuravlev, M. Muhin, op cit., p. 59. La somme de 500 roubles correspond au prix de vente moyen d'un tableau de Dejneka à la fin des années 1920, comme en témoignent les prix pratiqués par la commission d'achat du Glavnauka.

39. RGALI f. 2941, op. 1, d. 53. À défaut de présenter la première esquisse, l'artiste doit rembourser le premier versement dans un délai de deux mois. Si l'esquisse présentée ne convient pas, il est mis un terme à la collaboration sans que le peintre soit tenu au remboursement. Si la dernière esquisse proposée est refusée, le troisième versement n'est pas effectué.

40. Une seule esquisse est demandée dans ce cas. La première avance, $25 \%$ de la somme, est versée à la signature du contrat ; la deuxième, du même montant, est remise à la validation de l'esquisse, et le solde lors de la remise de la toile définitive. 
politiques et militaires, liés aux sujets des commandes. Dans le même ordre d'idée, à partir de 1935, on tend à faciliter l'accès des artistes aux bibliothèques, on leur présente des livres et des catalogues destinés à les aider dans leur travail. Dans le contrat de travail, la VOKS dit « mettre à disposition des peintres le matériel photographique nécessaire et donner des consultations sur les thèmes ». Généralement, dans le cadre de la préparation de ces grandes manifestations, les artistes peuvent également partir en «mission de création » afin « d'approfondir leur connaissance du sujet à traiter».

Il est bien difficile d'évaluer l'impact de ces mesures sur la création. On est loin des questions et des préoccupations artistiques et il est permis de s'interroger sur ce que les peintres peuvent tirer de ces manifestations. En outre, compte tenu des remarques concernant l'organisation des missions de création, nous doutons d'une mise en place rapide d'un tel système de conférences. Nous le voyons, un soin particulier est porté au sujet et à sa réalisation. En effet, une des grandes préoccupations dans l'entre-deux-guerres est de définir « ce qu'il convient de peindre ». Cela nous conduit à examiner de plus près la question du choix des sujets des commandes. Quels en sont les auteurs pour quels types de sujets ? La latitude dans le choix des sujets, déjà mise à mal au moment du Grand Tournant, se restreint sous la pression des jeunes artistes communistes fondateurs de la RAPH (Rossijskaja assocjacija proletarskih hudožnikov - Association russe des artistes prolétaires ${ }^{41}$, surtout à partir de la deuxième moitié des années 1930, après la réorganisation des institutions, la purge du Vsekohudožnik, et l'implantation en force d'anciens membres de l'AHRR aux postes décisionnaires importants.

\section{Le choix des sujets et l'application du plan thématique}

Il est malaisé de savoir qui, du peintre, du commanditaire ou des organisations socioprofessionnelles, décide réellement des thèmes. Selon les cas, chacun de ces acteurs peut se trouver à l'origine du sujet et/ou de l'iconographie de l'œuvre. En général, ils interviennent simultanément. Souvent le peintre s'adresse directement au commanditaire pour lui proposer ses services et un sujet plus ou moins défini. Tout comme on écrit à Stalin, les artistes sollicitent commandes, achats, missions directement des plus hautes instances. Les archives regorgent de pareilles requêtes à l'approche d'un grand événement ${ }^{42}$. En 1928, pour espérer une réponse favorable, les projets se doivent d'évoquer les principaux points du plan quinquennal. Les peintres adressent également leurs souhaits au MOSSH. Chargé de l'organisation des grandes expositions et notamment de la répartition des budgets, sous l'œil vigilant du NKP, il possède un pouvoir décisionnaire important. Les peintres composent eux-mêmes les comités d'expositions et influent sur l'éventail des sujets.

41. Cette association est l'équivalent de la RAPP (Rossijskaja Assocjacija Proletarskih pisatelej - Association russe des écrivains prolétariens), active dans le domaine littéraire.

42. RGALI, f. 2943, op. 1, d. 67. 
En 1934, c'est le MOSSH qui arrête le plan thématique des œuvres des différentes sections de l'exposition Oural-Kouzbass. La section du parti de l'Oural donne ensuite son approbation et, en dernier lieu, les artistes choisissent un thème dans la liste. Le MOSSH, comme le Vsekohudožnik, collabore ainsi avec divers organismes, industries ou kolkhozes locaux, et définit avec eux des sujets susceptibles de les intéresser afin d'orienter les thèmes des commandes. Dans les faits, le plan thématique se révèle largement flexible. Un article relatif à cette même exposition relate que, lors de leurs missions de création, les peintres ont signalé des sujets absents du plan, qui a été complété. Il a donc fallu augmenter la capacité d'accueil de l'exposition, présenter davantage de peintres et la date d'ouverture a dû être reportée ${ }^{43}$.

Sans parler ici de liberté totale de choix, il convient de mettre en avant une flexibilité relative dans l'application du plan thématique, souplesse qui tend à se restreindre après les réformes de 1936. L'usage du plan thématique devient alors plus systématique. Il semble que les administrations, comme le RVS ou le commissariat à l'Industrie lourde, prennent désormais une part de plus en plus importante à sa définition ${ }^{44}$. Pour l'exposition "L'industrie du socialisme », un comité de rédaction ad hoc est formé afin de préciser une série de thèmes à proposer au choix des artistes. Mais, en 1940, A. Solodovnikov ${ }^{45}$ revient sur la mise en place de cette manifestation. Il fustige les procédés en vigueur: les sujets «proposés, voire imposés » aux artistes ont émané de personnes sans lien direct avec le domaine artistique. Ce président-adjoint du Comité aux affaires artistiques propose deux améliorations au système de commande : les thèmes doivent être conçus pour être réalisables par des moyens picturaux et traités de manière artistique :

Dans certains cas, on a donné aux artistes une tâche qui, de manière générale, n'est pas réalisable en peinture. On exigeait, par exemple, de montrer comment le jeune Lenin étudie les classiques du marxisme. On peut écrire un livre à ce sujet, mais non peindre un tableau. On peut représenter Lenin un livre à la main, mais montrer, par des moyens picturaux, qu'il s'agit de l'étude du marxisme, c'est impossible. ${ }^{46}$

À cet effet, seules des personnes liées à la sphère artistique devraient être habilitées à intervenir à ce niveau. Pour autant, si l'implication des artistes eux-mêmes dans l'organisation est toujours souhaitable, il ne faut plus qu'ils soient contraints de se juger mutuellement. En outre, il apparaît indispensable que l'artiste puisse choisir un sujet qui corresponde à ses aspirations.

43. Voir Šim, « Donbass v živopisi » [Le Donbass en peinture], Bjulleten`'Vsekohudožnika, $\mathrm{n}^{\circ} 1-2,1935$.

44. Voir Cullerne Bown, op. cit., p. 95.

45. A. Solodovnikov, « $O$ vystavke 'Naša rodina' » [De l'exposition « Notre patrie»], Iskusstvo, $\mathrm{n}^{\circ} 2,1940$, p. 98.

46. Ibid. 
Le déroulement des commandes porte la trace des changements importants qui touchent les fonctionnaires et des réformes institutionnelles qui, après 1936, centralisent de plus en plus les décisions. Les anciens membres de l'AHR, qui monopolisent les postes à responsabilité, imposent davantage leur vision de l'art et leurs sujets de prédilection. Parallèlement, si le système semble bien avoir trouvé des commanditaires (et acheteurs), ceux-ci, sans doute amenés à tirer profit de la situation de dépendance économique des artistes, tendent à orienter de plus en plus l'iconographie par des formulations très éloignées des préoccupations artistiques. Cette évolution générale vaut pour l'engagement sous contrat.

\section{L'engagement sous contrat : entre commande et mécénat}

Le système d'engagement sous contrat se met en place à partir de 1929, d'abord au sein des éditions de l'AHR ${ }^{47}$, puis de l'Izogiz en $1930^{48}$, et enfin du Vsekohudožnik à partir de 1932. En 1930-1931, 74 artistes travaillent ainsi pour l'Izogiz. Le Vsekohudožnik débute très timidement, engageant 4 artistes en 1932, 34 en 193349, puis 150 en $1935^{50}$ contre 116 pour l'Izogiz la même année ${ }^{51}$. Lors de la fondation du MOSSH, en 1932, le secteur pictural de l'Union des artistes de Moscou compte 478 membres.

Il nous est difficile, aujourd'hui, de cerner l'évolution du système d'engagement sous contrat dans la deuxième moitié des années 1930, c'est-à-dire après l'arrestation et la mort du responsable du Vsekohudožnik, Jurij Slavinskij, et le démantèlement de l'institution. La reconstitution proposée ici repose essentiellement sur l'étude du fonds d'archives de l'AHR et de la presse : les organes de l'AHR puis de la RAPH, entre 1929 et 1932 (Iskusstvo v massy et Za proletarskoe iskusstvo), Bjulleten' Vsekohudožnika, et les revues spécialisées des années 1930 (Tvorčestvo et Sovetskoe iskusstvo).

\section{3-1 Les modalités du contrat}

L'artiste s'engage à livrer un certain nombre d'œuvres dans l'année contre un salaire mensuel. Cette rémunération est comprise entre 200 et 300 roubles à l'Izogiz en $1931^{52}$, et entre 250 et 500 roubles au Vsekohudožnik pour deux ou trois

47. Zernova, op. cit., p. 71 .

48. V. Stukov, «Pljusy i minusy kontraktacij » [Avantages et inconvénients de l'engagement sous contrat], Za proletarskoe iskusstvo, $\mathrm{n}^{\circ} 3-4,1931$, p. 28.

49. Vsekohudožnik, M., 1934.

50. Slavinskij, art. cit., p. 4.

51. RGALI, f. 2943, op. 1, d. 1134, Protokoly zasedanija bjuro sektora živopisi, 29.I28.XII.1935 g., 1. 17-18.

52. Zernova, op. cit., p. 71. 
tableaux par an ${ }^{53}$. (Rappelons que le salaire moyen d'un ouvrier de la capitale est alors de 120 à 140 roubles; de 240 roubles en 1935). En 1934, le Vsekohudožnik propose trois niveaux de salaire : 300, 400 ou 500 roubles pour un ou deux travaux ${ }^{54}$. La rétribution est fonction de la cote de l'artiste. En outre, les meilleures œuvres sont censées recevoir des prix. Mais les choses sont moins simples qu'il n'y paraît.

Le contractant accorde au Vsekohudožnik un droit préférentiel sur l'achat et l'exposition de la production de cette année-là. Les tableaux sont d'abord estimés : le peintre et la coopérative doivent s'entendre sur le prix de chaque toile. Si, au final, le Vsekohudožnik choisit des œuvres dont le montant dépasse la rétribution initiale, il procède à un réajustement. Si, inversement, ce montant est inférieur à la rétribution perçue par l'artiste, un accord complémentaire est conclu. Dans le cadre de ces contrats, les œuvres deviennent la propriété de la coopérative et/ou de la commission d'achat d'État; en cas de reproduction, l'artiste conserve les droits d'auteur.

Au sein de l'Izogiz, les tableaux sont généralement exclusivement réservés à la reproduction et à la diffusion de masse. Certains artistes s'insurgent même contre le fait que les originaux, rendus inaccessibles, ne soient jamais exposés ${ }^{55}$. Le phénomène est tellement répandu que, dans le cadre des réflexions sur l'organisation de la nouvelle Académie des artistes, il suscite des questions sur le statut de l'artiste et sa formation $^{56}$.

Le principe de l'engagement sous contrat rejoint ainsi celui du mécénat, mais l'idée d'orienter le travail sur des thématiques politiques rapproche ce type de mécénat d'un système de commande. Il ne faut cependant pas se limiter à ce constat et il convient d'examiner ce qu'il en est en pratique. Quels sont les sujets imposés ? Les artistes répondent-ils à ces demandes, et si oui, comment ? D'autant plus que des problèmes organisationnels ou inhérents à la personnalité des auteurs peuvent survenir entre le projet initial et sa mise en œuvre.

\section{3-2. Du choix du sujet au tableau fini}

Dans le domaine éditorial, l'Izogiz définit assez précisément le sujet confié à l'artiste. En 1934, le Vsekohudožnik indique qu'un seul thème général est proposé aux peintres, à partir duquel chacun peut élaborer ses sujets : «Bilans du Premier

53. Vsekohudožnik, M., 1934 et RGALI f. 2943, op. 1, d. 1134. Protokoly zasedanija bjuro sektora živopisi, 4.III.1935 g.

54. Slavinskij, art. cit., p. 15

55. Ju. Slavinskij, «Plenum Federacii » [Le plénum de la Fédération], Brigada hudožnikov, $\mathrm{n}^{\circ} 2,1931$, p. 12.

56. RGALI f. 2941, op. 1, d. 135, Protokoly zasedanij komissii po organizacij Akademij hudožnikov, $1.23 \mathrm{sqq}$. Une des questions principales est de savoir si l'artiste qui peint uniquement en vue de la reproduction demeure un artiste peintre ou s'il entre dans la catégorie des graphistes. 
Plan quinquennal, à l'assaut du Deuxième Plan quinquennal $»^{57}$. Si l'orientation politique est évidente, l'intitulé demeure vaste et flou. De plus, il semble que les artistes ne prennent celui-ci qu'à titre indicatif, sans y voir une obligation. En témoignent, par exemple, les œuvres réalisées en Crimée par Dejneka (ill. 3) ou la Femme dans un hamac (ill. 4) de Pimenov.

Un suivi de la production artistique est organisé, notamment par le biais du contrôle des esquisses. Mais une série de dysfonctionnements frappe cette organisation. En 1931, au cours du bilan des engagements sous contrat de l'Izogiz, un article souligne que les artistes, qui devaient rendre dix tableaux et dix esquisses, ne se sont même pas acquittés de la moitié de leur tâche. Selon l'auteur, les sujets n'ont pas été donnés à temps, la consultation politique ${ }^{58}$ s'est révélée trop faible, les artistes sont partis tardivement sur le terrain, les collectifs de création se sont formés au hasard, et le contrôle du travail des artistes sous contrat était inexistant ${ }^{59}$. Après la purge qui touche les éditions d'art d'État en 1931, un contrôle strict se met en place. Des rédacteurs suivent et encadrent le travail des peintres. En 1936, Liušin se plaint d'ailleurs de leur action, qu'il qualifie de «tutelle mesquine conduisant à l'illustration ${ }^{60}$. Le Vsekohudožnik soulignera plus tard ses propres difficultés de contrôle du travail des peintres ${ }^{61}$. À partir de 1935, il tente de mettre en place une consultation plus active sur les questions politiques, organise l'approvisionnement en matériel nécessaire au travail et constitue une photothèque. Tous ces efforts ne semblent aboutir que partiellement et n'avoir qu'un impact limité sur la création. Ce constat dressé en 1936, à la suite de l'exposition des artistes engagés sous contrat l'année précédente, en fait foi :

Moins de la moitié des artistes représentés à l'exposition étaient sous contrat. Sur un total de presque 500 œuvres soumises au jury, près de la moitié a été éliminée. Les trois quarts des œuvres proposées relevaient du paysage, une part significative du portrait, et à peine $15 \%$ d'une thématique idéologique.

Dans n'importe quel autre domaine, en URSS, de tels résultats auraient été qualifiés de grave incident [...]. Mais sur le front des arts plastiques, cela n'a pas eu l'air de produire un quelconque effet. Personne ne s'est soucié de savoir qui sont précisément ces incapables et ces gens qui gâchent leur travail. Et non seulement le Vsekohudožnik, mais aussi le MOSSH, font mine de n'y être pour rien.

La majorité des artistes sous contrat a fourni, par exemple, en guise de sportifs, des paysages d'automne, d'été, et des fleurs. En guise d'agriculture, on a eu à nouveau des fleurs, mais cette fois des fleurs des champs, puis des fruits à la place d'un petit-déjeuner de kolkhozien, ou encore une petite-bourgeoise

57. Ibid.

58. Des spécialistes de différents domaines dispensent aux artistes des informations d'ordre politique, économique ou social, afin que ceux-ci traitent « fidèlement » leur sujet.

59. Malkin, « Kontraktacija hudožnikov rasširjaetsja » [L'engagement sous contrat des artistes prend de l'ampleur], Sovetskoe iskusstvo, 22 janvier 1931.

60. Aleksandr Ljušin, Avtobiografija [Autobiographie], M., 1936, p. 181-182.

61. Slavinskij, « Novaja kontraktacija », p. 15. 


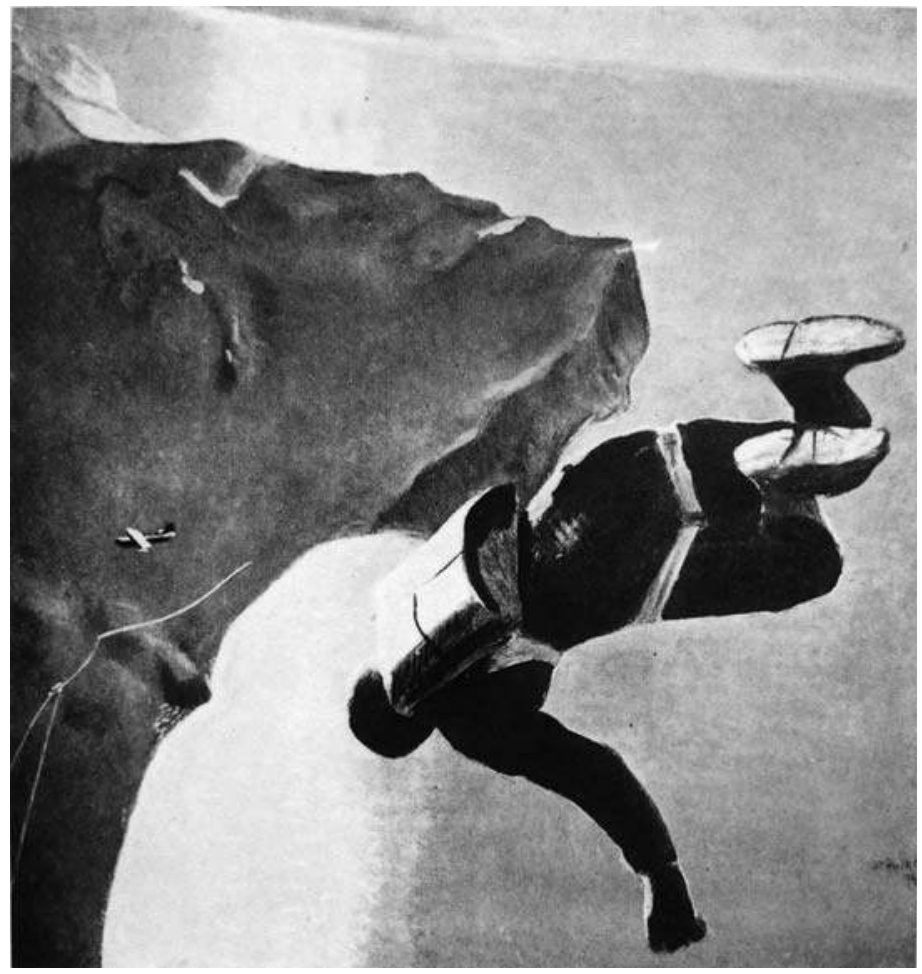

Illustration 3. Aleksandr Dejneka, Parachutiste au-dessus de la mer, 1934, huile sur toile, $174 \times 122 \mathrm{~cm}$, Musée national du Kirghizistan, dr.

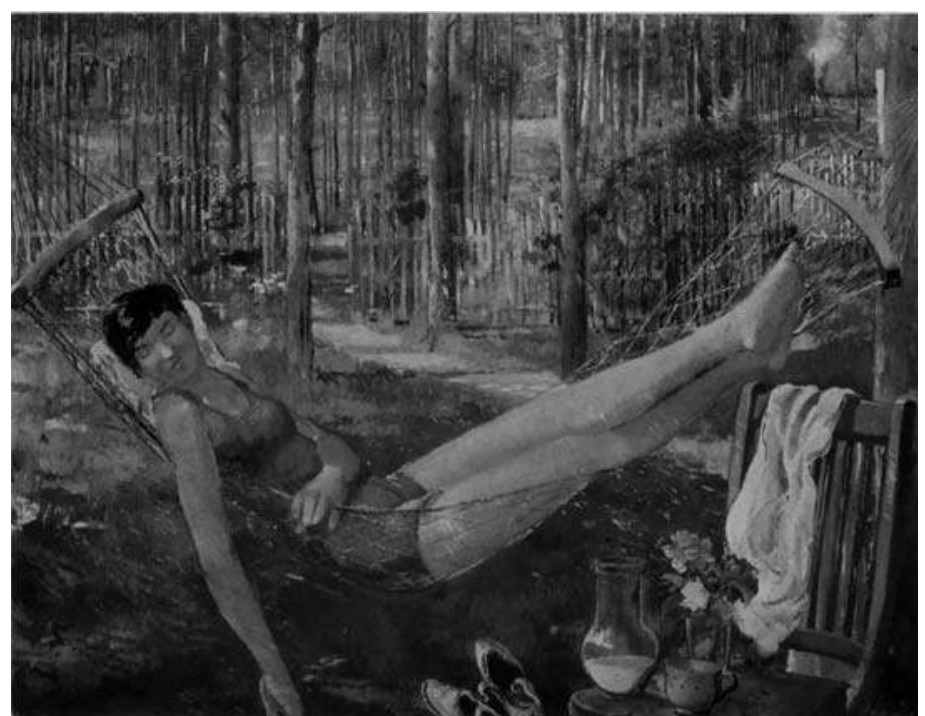

Illustration 4. Jurij Pimenov, Femme dans un hamac, 1934, huile sur toile, $129 \times 161 \mathrm{~cm}$, Musée russe, Saint-Pétersbourg, dr. 
flasque avec un petit air frivole. Au lieu d'un sujet kolkhozien, encore et toujours une journée d'automne, etc. Personne n'a rien proposé concernant les pionniers, excepté deux artistes. ${ }^{62}$

Le catalogue de l'exposition témoigne de cet état de fait. Le Vsekohudožnik y prône l'intérêt et l'utilité de genres comme le paysage que certains artistes et critiques veulent voir disparaître au profit d'une peinture à contenu idéologique. Rappelons qu'une fois en possession des œuvres, le Vsekohudožnik les expose et les revend. De ce point de vue, il est très probable qu'il ne parvienne effectivement pas à orienter la production picturale vers des œuvres thématiques, par suite de défauts d'organisation et de la résistance des artistes, ou qu'il n'ait tout simplement pas l'ambition de promouvoir des sujets spécifiquement soviétiques, ceux-ci n'étant pas les plus demandés. Ces deux cas de figures sont à prendre en considération.

Pas plus que les commandes, l'engagement sous contrat ne peut, à lui seul, assurer un minimum vital aux artistes. C'est la conjonction de ces différents systèmes ainsi que les activités parallèles (autres pratiques artistiques, enseignement, travail rémunéré dans les institutions, participation aux jurys d'exposition, vente, reproduction des tableaux, etc.) qui permettent à l'artiste de vivre de son art. À cela s'ajoute un aspect fondamental du système soviétique stalinien : les privilèges et autres apports en nature distribués par l'État à certains peintres en faveur (nourriture, vêtements, appartements, sommes complémentaires, datchas, voitures, accès gratuit à certains restaurants et lieux de villégiature, etc.). Enfin, l'engagement sous contrat, comme la commande, se combine généralement avec une mission de création. Aleksandr Dejneka réalise ainsi sa remarquable série sur les parachutistes lors d'une mission en Crimée auprès des aviateurs de l'Armée rouge. Ce voyage s'effectue dans le cadre de son engagement sous contrat avec le Vsekohudožnik en 1934.

\section{4. « Les missions de création »}

Dans les années 1920-1930, les missions de création sont extrêmement courantes. Elles permettent aux artistes de se rendre dans n'importe quelle partie du territoire soviétique, mais également à l'étranger. Leur durée, variable au début, se réduit au fil du temps : les missions intérieures qui étaient d'un ou deux mois dans les années 1920, n'excèdent que rarement quinze jours à partir du milieu des années 1930 . Dans les années 1930, le peintre part en mission notamment (mais pas nécessairement) pour exécuter une commande en vue d'une exposition ou honorer un engagement sous contrat. De nombreuses institutions et organisations commanditent les missions sur leurs propres fonds ou en obtenant des subsides de l'État. Le SNK, le NKP, le Glavnauka puis le Glaviskusstvo, l'AHRR, le MOSSH et le Vsekohudožnik subventionnent ces voyages particuliers. Ainsi en 1931, 113 peintres sont

62. N. Nikolaev, « Kontraktacija hudožnikov 1935 goda » [L'engagement sous contrat des artistes en 1935], Tvorčestvo, ${ }^{\circ}$ 8, 1936, p. 13-17. 
commandités sur les fonds du SNK ${ }^{63}$. En 1930, dans un contexte de réformes institutionnelles et de centralisation, une commission interministérielle chargée de centraliser les informations relatives à ces missions et d'intervenir dans la répartition des budgets est créée au sein de le secteur des arts du NKP.

Le commanditaire doit pourvoir à un certain nombre de besoins. Il prend en charge les frais de déplacement (billet de train aller-retour), d'hébergement et de nourriture (500 roubles pour ce dernier poste dans les années 1920). À cela peut s'ajouter une allocation en nature pour l'équipement : chaussures, tenues de travail ou autres objets courants ${ }^{64}$. À partir de la fin de l'année 1933, les documents d'archives mentionnent un mode de rémunération différent ${ }^{65}$. Les sommes versées sont globalement plus importantes et la cote du peintre entre en ligne de compte. Les artistes sont répartis selon deux catégories, en fonction de leur qualification et de l'éloignement de leur destination, ce qui explique les disparités que nous avons pu constater dans le montant des sommes allouées : 1500 roubles pour un groupe et 2000 roubles pour un autre. Dans tous les cas, les œuvres demeurent la propriété des artistes.

Si les déplacements individuels semblent fréquents jusqu'au début des années 1930 , la forme du voyage en brigade ${ }^{66}$ tend à se répandre par la suite, sans jamais parvenir à s'imposer totalement. Parallèlement, la durée du séjour se réduit et les périodes semblent établies selon la nature des lieux (agricole, industriel ou chantier de construction de voies de communication). Le voyage strictement encadré et étroitement surveillé dans des lieux vitrines ne s'impose pas d'emblée. Qui choisit alors les destinations et les thèmes? Selon quels critères? Quelles sont les conditions de séjour de l'artiste?

\section{4-1. Le choix de la destination et des thèmes}

Pour les années 1920, trois exemples nous permettent d'observer des variantes de missions intérieures.

63. RGALI f. 645, op. 1, d. 465, Perepiska sektora iskusstv Narkomprosa RSFSR s Sovnarkomom, Narkomfinom i drugimi o komandirovkah hudožnikov po SSSR, zajavlenija i otčëty hudožnikov o komandirovkah, 1. 47.

64. RGALI f.645, op. 1, d.440, Protokoly zasedanij mežduvedomstvennoj komissij po otpravke hudožnikov v komandirovki, zajavlenija hudožnikov o predstavlennih komandirovok, perepiska hudožestvennyh obščestv s sektorom iskusstva Narkomprosa RSFSR, 9 maja 1931 g. - 17 dek. 1931g., 1. 16, 21.

65. RGALI f. 2943, op. 1, d. 18, Dokumenty po tvorčeskim komandirovkam, 9.V-11.XII.1933 g., 1. 57.

66. La brigade se compose, en moyenne, de trois à cinq artistes et peut rassembler différents praticiens (peintres, sculpteurs, dessinateurs, etc.). Plusieurs brigades peuvent être envoyées au même endroit. 


\section{Les années 1920}

En 1925, Kliment Red’ko part dans la région de Mourmansk poursuivre ses recherches sur le rendu de la lumière. Il s'attache plus particulièrement à l'étude de phénomènes, telle l'aurore boréale, dont un scientifique scandinave a percé le secret au début du $\mathrm{XX}^{\mathrm{e}}$ siècle. Cette mission permet à l'artiste de développer ses recherches abstraites, baptisées «électro-organisme », sur le terrain de la peinture figurative. Le peintre a délibérément choisi sa destination, en accord avec ses préoccupations artistiques. Mais cette liberté de choix est-elle systématique ? Rien ne permet d'affirmer qu'en 1924 Dejneka se rend de sa propre initiative dans les mines du Donbass. Il est commandité par le journal $U$ stanka, pour lequel il réalise nombre d'illustrations. Mais ses observations, ses croquis, en somme le matériau artistique amassé lors de ce voyage, vont également servir à élaborer des toiles, comme par exemple Avant de descendre dans la mine. L'artiste met ainsi à profit une mission effectuée dans un certain cadre pour développer ses réflexions picturales. En outre, nous connaissons son attrait pour le monde ouvrier.

Un dernier point retient notre attention. Il s'agit de la manière dont procède l'AHRR dans le cadre de l'organisation de diverses manifestations. Considérons l'exposition «Vie et quotidien des peuples de l'URSS » en 1926. Grâce à des subventions allouées par le NKP ou le SNK, l'AHRR envoie certains de ses membres en mission. Le choix des destinations paraît cette fois s'effectuer en fonction des sujets retenus afin que les créateurs puissent inscrire leur travail dans le thème de l'exposition. Les missions comportent déjà un caractère plus ou moins marqué de reportage, conformément aux positions artistiques du groupe. L'AHRR interprète ici, à sa manière, la tradition du voyage d'étude des peintres Ambulants ${ }^{67}$. Il s'agit pour l'Association de renoncer à l'habituel séjour estival ${ }^{68}$, durant lequel l'artiste s'applique surtout à la réalisation de paysages, au profit de missions orientées vers le monde ouvrier et paysan avec ses usines et ses kolkhozes ${ }^{69}$. Les expériences de l'AHRR vont servir de modèles, et les jeunes militants communistes, futurs membres de la RAPH, qui tentent d'évincer la vieille intelligentsia russe au moment du Premier Plan quinquennal, vont radicaliser cette conception des missions de création et tenter de l'imposer à tous les peintres.

67. Voir E. Nesterova, Les Ambulants, SPb. : Aurora, 1996, p. 13. Les Ambulants avaient pour habitude de se rendre, l'été, dans leur région natale pour peindre. Les plus démunis se contentaient d'une remise dans les environs de Saint-Pétersbourg.

68. En 1932, il est question d'organiser également des missions tout au long de l'année. Ce qui sera fait. Voir « Zadanija zaveršajuščego goda », Brigada hudožnikov, n 1, 1932, p. 50.

69. Voir T. Gaponenko, «Zovy žizni » [L'appel de la vie], Iskusstvo v massy, n 5-6, 1929, p. 11. 


\section{9-1932 : les missions comme outil de " rééducation des compagnons de route » (perevospitanie popučikov)}

Dans les années 1928-1931, la jeunesse communiste s'impose par la force ${ }^{70}$. Dans le domaine de la peinture, ces jeunes membres du parti noyautent l'Association des artistes de la Russie révolutionnaire, et finissent par fonder la RAPH, en 1931. À coups de déclarations tonitruantes dans leurs organes de presse (Iskusstvo $v$ massy puis Za proletarskoe iskusstvo), ils tentent d'imposer leur approche de l'art. Avant tout, ils prônent une peinture compréhensible par les masses, politisée, dont les sujets relèvent exclusivement d'une « thématique soviétique ». En outre, ces jeunes communistes dénoncent les peintres qu'ils considèrent comme des artistes de droite et des popučiki [compagnons de route]. Le terme devient alors péjoratif et désigne non seulement les peintres abstraits, mais aussi des peintres importants de la figuration russe des années 1920, membres de groupes majeurs, tels que les Quatre-Arts ou la Société des artistes de chevalet.

La jeunesse communiste investit les organisations socioprofessionnelles et le NKP. Elle entend utiliser les missions de création comme moyen de « rééduquer » les peintres dénoncés comme « compagnons de route ». Ces voyages dans les hauts lieux de l'édification industrielle et kolkhozienne ${ }^{71}$ devaient inciter les créateurs à se départir de leurs sujets habituels au profit de la thématique soviétique. Selon les futurs membres de la RAPH, le peintre doit connaitre la vie pour l'exprimer, en témoigner et informer les masses, ses tableaux ont pour fonction de promouvoir et de faire connaître l'ampleur des grands projets. Le climat de ces années du Premier Plan quinquennal se révèle particulièrement lourd, les futurs membres de la RAPH étouffent toute vie artistique. Cependant, leurs conceptions ne sont pas toutes appliquées immédiatement et totalement, fort heureusement. Leur action se heurte à la réalité du terrain, au manque de moyens, à la relative désorganisation inhérente aux réformes institutionnelles, et enfin à la résistance de nombreux peintres. Quelques exemples feront mieux comprendre le fonctionnement effectif de ces missions.

\section{Une ambition à l'épreuve des faits}

Dès sa création, en 1928, le Vsekohudožnik organise des missions dont les artistes choisissent les lieux ${ }^{72}$. En 1929, le NKP (Glaviskusstvo) organise les premières

70. Voir les travaux sur la « Révolution culturelle », notamment ceux de S. Fitzpatrick, op. cit.

71. Voir Katalog vystavki rabot hudožnikov komandirovannyh v raionah industrial'nogo i kolhoznogo stroitel'stva [Catalogue de l'exposition des travaux des artistes envoyés dans les régions de l'édification industrielle et kolkhozienne], M. : 1932. Maslennikov souligne par exemple, au sujet de Bogaevskij, que son voyage à Bakou et l'observation des puits de pétrole n'ont rien changé à sa façon de représenter le paysage. À ses yeux, un changement de sujet doit obligatoirement entraîner une métamorphose du style. On pense alors que le contact avec la nature, avec la vie, va faire de ces artistes des auteurs engagés, impliqués dans la thématique soviétique.

72. S. Lučiškin, Ja očen' ljublju žizn’ (Stranicy vospominanja), M., Sovetskij hudožnik, 1988, p. 122. 
missions vers les régions de l'édification industrielle et kolkhozienne ${ }^{73}$. En 1930, le SNK publie un décret sur les «missions dans les régions industrielles, les kolkhozes et les sovkhozes $\gg^{74}$. Pourtant, en 1931 le système n'est toujours pas opérationnel. Le syndicat des professions artistiques (Profsojuz rabotnikov iskusstv, Rabis) déplore la quasi-absence de plan de répartition des envoyés en mission selon les régions, ainsi que celle de thèmes pour guider le travail des artistes. En effet, en 1932, Nikolaj N. Maslennikov, responsable de la nouvelle commission, rapporte que, l'année précédente, la majorité des artistes s'est précipitée dans les stations balnéaires du bord de la mer Noire, négligeant ainsi des sites caractéristiques de la construction socialiste ${ }^{75}$ et ce, faute de plan préétabli.

Le manque de cohérence rend impossible toute utilisation des œuvres dans le cadre d'expositions thématiques. Dans le texte, le syndicat insiste pour qu'un effort d'organisation soit consenti, mais aussi sur le fait que les tâches doivent être attribuées aux artistes en accord avec leurs aspirations créatrices individuelles. Il défend également la possibilité d'une expression large et originale ${ }^{76}$. La détermination préalable des destinations et des thèmes de travail, qui doit s'effectuer par l'intermédiaire du secteur des arts plastiques du $\mathrm{NKP}^{77}$, se heurte cependant à un ensemble de difficultés : inertie des interlocuteurs, divergences d'opinion quant à la définition même des lieux importants ${ }^{78}$ et surtout dysfonctionnements des jeunes institutions encore peu et mal organisées.

Le NKP tente donc désespérément de centraliser les informations relatives aux missions organisées par les différents commanditaires : Izogiz, MOSSH, Vsekohudožnik, Mossovet, etc. En 1931, mis à part l'Izogiz, aucun organisme n'a fait parvenir de listes complètes. Chacun s'arrange dans son coin, faisant fi des sollicitations répétées du NKP qui peine à structurer un plan thématique préalable à l'envoi en mission. En juin, alors que les départs approchent, la répartition des artistes est loin d'être fixée. Les listes de commandités mentionnent rarement la destination et, plus exceptionnellement encore, le thème du travail. Il faut dire qu'à l'approche de l'été, les fonctionnaires eux-mêmes partent en vacances et personne n'est affecté, de manière sérieuse, au suivi des départs en mission ${ }^{79}$.

73. V.S. Manin, op. cit., p. 139. L'auteur indique que près de 70 peintres partent cette année-là, contre 200 en 1930.

74. Nous ne sommes malheureusement pas parvenus à retrouver ce document. Seule Anikeva, dans son ouvrage sur Aleksandr Pahomov, en cite un extrait. Les artistes sont envoyés « pour étudier à la fois l'économie (outillage, exploitation) et le byt (quotidien) des masses ». Voir V. Anikieva, A.F. Pahomov, L. : Leningr. oblast. Sojuza sovetskih hudožnikov, 1935, p. 35.

75. Cf. Maslennikov, Katalog vystavki rabot hudožnikov komandirovannyh..., p. 2.

76. RGALI f. 645 , op. 1, d. 465, 1. 24.

77. Sovetskoe iskusstvo, 23 mai 1931.

78. RGALIf. 645, op. 1, d. 440,1. 17-18.

79. RGALI f. 645, op. 1, d. 440, 1. 16-18. L'enquête sur le travail de la commission interministérielle déléguée aux missions de création dans les régions de l'édification industrielle et kolkhozienne rapporte les faits suivants : «Parti en vacances, le président de la commission interministérielle, le camarade Brajlovskij, a été remplacé par le camarade Budilov, lui-même parti avec les commandités du premier tour. Ce dernier a été remplacé par le camarade Nikolaevič, parti également en mission peu de temps après, etc. ». 
Pareil laxisme a deux conséquences. En premier lieu, il est vraisemblable que les artistes parviennent à jouer de cette désorganisation afin de choisir plus librement leur lieu de travail. En 1931, Aleksandr Labas profite d'une mission pour se rendre auprès de son frère, commandant de l'Armée rouge dans le Caucase. Compte tenu du poste de celui-ci, le déplacement a probablement été relativement simple à obtenir ${ }^{80}$. Cette même année, Ekaterina Zernova en profite pour visiter des parents éloignés ${ }^{81}$. Enfin, au-delà de l'aspect familial, le choix de ces destinations permettait certainement de limiter les frais courants sur place, d'hébergement notamment. Plus tard, en 1934, Dejneka se rend en Crimée auprès des aviateurs de la marine et prend part à leur vol d'entraînement. Cette destination correspond à sa passion pour l'aviation et ses recherches plastiques dans le rendu des sensations en vol. Certains artistes parviennent ainsi, en quelque mesure, à influer, voire à choisir, leur destination en fonction de préoccupations familiales et personnelles ou de recherches artistiques, tout en tenant plus ou moins compte des demandes thématiques. Il demeure plus délicat d'établir le lien entre David Sterenberg et le kolkhoze où il se rend pour travailler sur la récolte du thé, ou entre Sergej Lučiškin et ses destinations en 1930, 1931 ou 1936 : il séjourne successivement dans un kolkhoze au bord du fleuve Kouban, dans un camp militaire de la région Tcherkasskaïa ${ }^{82}$, en Arménie enfin.

Progressivement, la formulation des sujets et thèmes de travail se précise. En 1931 , le contrat type ${ }^{83}$ comporte une rubrique pour la destination et la mention d'une thématique ainsi commune à tous : «l'URSS ». Le sujet est légèrement affiné au point suivant : « À l'assaut de la 3 e année du plan quinquennal ! ». Les indications larges et floues sont parfois précisées, au cas par cas. Pavel Kuznecov part ainsi étudier «la production pétrolière à Bakou » ${ }^{84}$. Nous supposons que la définition préalable du thème se généralise progressivement.

Si la première conséquence du désordre organisationnel est une marge de manœuvre dans le choix des thèmes et des destinations, le second effet se révèle tout à fait néfaste. Nombre d'artistes sont contraints de rester sur place ${ }^{85}$ soit parce que les financements ne leur parviennent pas, soit parce que personne n'est en mesure de prendre une décision et de les informer de leur destination. Il arrive encore fréquemment que la somme allouée ne leur soit jamais versée en totalité. Il

80. «En 1931, je suis envoyé en mission pour travailler dans l'armée. Je n'avais pas eu de contact avec elle depuis 1920-1921, date de mon retour du front Est, après la défaite de Kolčak. Aussi, je me réjouissais de cette mission. Mon frère aîné était chef de régiment, en Ukraine. Ayant appris que je devais effectuer ma mission dans une section militaire en manœuvres, il m'a invité à le rejoindre dans son secteur ». Voir A. Labas, «Iz Vospominaja », Tvorčestvo, $\mathrm{n}^{\circ} 7,1978$, p. 17-18.

81. Zernova, op. cit., p. 75.

82. Envoyé là par le PUR durant l'été 1931. Voir RGALI f. 2942, Hudožestvennye organizacijpredšestvenniki MOSSHa, op. 1, d.23, Otčët hudožnikov o tvorčeskih komandirovkah po SSSR za 1931 g., 1. 23-24.

83. RGALI f. 645, op. 1, d. 440,1. 16.

84. Ibid.

85. RGALI f. 645, op. 1, d. 440,1. 17. 
faut ajouter à cela des difficultés à s'approvisionner en matériel ${ }^{86}$. Enfin, certains artistes partent avec un retard plus ou moins important. Dans tous les cas, il leur est difficile de présenter les œuvres à temps. Ils demeurent parfois longtemps dans l'expectative et se rendent quotidiennement aux nouvelles auprès de l'organisme commanditaire. Leur travail s'en trouve paralysé.

Au tournant des années 1920 et 1930, le voyage semble revêtir deux significations contradictoires. Considéré comme un moyen de travailler, il est à ce titre très recherché des artistes qui sollicitent le NKP et les organismes commanditaires pour être inclus dans les listes des élus, - selon le même procédé que pour les commandes, les lettres adressées par les peintres comportent des propositions de destination et de thèmes de travail ${ }^{87}$. Pour les commanditaires, ces séjours répondent à l'objectif de « rééducation » politique et artistique des missionnés, dont les effets devraient apparaître au niveau du choix des sujets et dans le changement des références artistiques. Les missions intérieures acquièrent un double rôle paradoxal : elles constituent un moyen de subsistance tout en s'intégrant dans un contexte de volonté de mise au pas artistique. Cette dernière ambition se trouve encore freinée par la manière dont se déroulent les séjours.

\section{4-1. Les conditions de voyage}

Considérons toujours la situation au tournant des années 1920 et 1930, lorsque le NKP initie les commandes. Avant de s'attarder sur le déroulement du séjour, précisons quelques données concernant sa préparation.

À partir de 1930, dans l'optique de rééduquer les « compagnons de route », le contrat contraint l'artiste à assister à des séminaires politiques avant son départ. La FOSH (Federacija ob"edinenij sovetskih hudožnikov - Fédération des unions artistiques soviétiques) gère le programme et l'organisation. Ces exposés sur «l'industrialisation du pays et le plan comme méthode économique » ou «la collectivisation agraire ${ }^{88}$ sont censés aider l'artiste à mieux appréhender la réalité du terrain qu'il s'apprête à découvrir. Il est malaisé de déterminer l'impact de ce type de discours sur la vision de l'artiste. D'ailleurs, compte tenu de la précipitation dans laquelle les missions de 1930-1931 se mettent en place, certains artistes se voient exemptés de ce pensum. Là encore, la structure de ces séminaires s'établit progressivement.

Le discours sur la pratique artistique se situe essentiellement au niveau de la réception. D'un côté, la critique fait son travail d'évaluation des résultats. De l'autre, les artistes participent à des soirées de création (tvorčeskie večera), débats

86. Nous pensons notamment aux problèmes engendrés par la production de toiles de trop grand format, inadaptées au déplacement. Voir L. Bačinskij, «O hudožestvennyh kraskah » [À propos des couleurs des peintres], Sovetskoe iskusstvo, 15 septembre 1932.

87. RGALI f. 645, op. 1, d. 440,1. 81-98.

88. RGALI f. 645 , op. 1, d. 440,1. 50-52. 
houleux au cours desquels ils discutent et analysent l'œuvre d'un peintre déterminé dans le but officiel de corriger, de condamner les erreurs et d'encourager les réussites. Cette pratique, aux allures de procès, entretient de violentes rivalités entre les peintres et ne se limite pas à l'appréciation des travaux rapportés des missions.

L'ensemble des préliminaires au voyage suit un schéma que nous retrouvons sur le terrain de la commande. Qu'en est-il alors de la situation des artistes sur place et de leurs obligations afférentes au séjour? Les peintres sont invités à s'informer pour choisir précisément les lieux de visite, prendre connaissance des thèmes et des photographies. Ils doivent également entrer en rapport avec les organisations locales (usine, kolkhoze, armée...) qui les accueillent. Les conditions de vie et de travail de l'artiste sont ainsi fonction de l'intérêt que lui portent le responsable et le personnel local. À ce propos, la période estivale pose de sérieux problèmes : en effet, quelle attention le responsable d'un kolkhoze peut-il prêter à un artiste à l'époque des moissons? En outre, le peintre est chargé d'accomplir sur place un «travail d'intérêt social» auprès des ouvriers, kolkhoziens ou gardes rouges. Celui-ci peut prendre des formes diverses et variées : affiches, journaux muraux, expositions, conférences, cours de dessin, etc. Or, là encore, l'artiste reste confronté au problème des moissons. Lučiškin mentionne en effet qu'aucune activité n'a pu être menée dans le kolkhoze où il était envoyé, la population étant indisponible, mobilisée par les récoltes ${ }^{89}$.

\section{Conclusion}

Des points communs apparaissent entre le fonctionnement des commandes, de l'engagement sous contrat et des missions de création. La mise en place des modes de contrôle et du suivi de la création des artistes se révèle tout autant progressive et chaotique. Les organisations socioprofessionnelles et les peintres affichent leur rôle prépondérant dans la définition des sujets et du contenu des œuvres. En plaçant ainsi les créateurs aux postes décisionnaires, le pouvoir s'appuie sur les rivalités entre les peintres et les exacerbe. Chacun tente d'imposer sa conception de l'art. Enfin, la mise en œuvre de directives volontairement floues génère des pratiques. C'est ainsi que plusieurs spécificités du système soviétique ressortent dans le champ artistique. Chacun des types de contrat étudiés s'impose à la fois comme soutien à la création et système contraignant.

On ne saurait voir désormais dans la période 1928-1932 une rupture franche, il faut l'appréhender dans sa complexité, avec ses nouveautés et son héritage. Il convient également de prendre en considération l'interprétation de la réalité environnante par l'artiste.

Un prolongement possible du présent travail pourrait s'inscrire dans une perspective comparatiste. Une ouverture interdisciplinaire amène à confronter les systèmes de commande effectifs dans les domaines des arts plastiques et de la

89. RGALI f. 645 , op. 1, d. 465, 1. 8-9. 
littérature : de nombreuses disciplines recourent aussi aux missions de création. Une comparaison avec d'autres pays ayant mené une politique interventionniste dans la sphère artistique après la crise de 1929, sans se limiter à l'Allemagne nazie ou à l'Italie fasciste, représente également une piste intéressante. Nous pensons notamment à des rapprochements avec certaines entreprises du New Deal ou avec les mesures ayant conduit à la création du $1 \%$ en France ${ }^{90}$. Enfin, l'étude des liens entre les systèmes de commande soviétiques et le fonctionnement du marché de l'art dans la Russie tsariste constitue un champ d'investigation à envisager.

EHESS, Centre d'études des mondes russe, caucasien et centre-européen

cecile.pichon-bonin@club-internet.fr

90. Le New Deal est mis en place en 1932 et le Federal Art Project entre 1935 et 1939. Ce programme établit le principe de subventions régulières allouées aux artistes au chômage. Les commandes gouvernementales apparaissent comme un moyen de donner du travail aux artistes, et l'accent est mis sur la peinture murale ou la décoration d'édifices publics. Beaucoup de peintres de genre ont bénéficié de ce programme, la plupart peignaient dans un style plus ou moins réaliste. En France, l'idée de consacrer un pourcentage du coût des constructions publiques à la « décoration monumentale » apparaît en 1936 à travers deux projets de loi. Là encore, il s'agit de donner du travail aux artistes au chômage. Cependant, ces projets n'aboutiront pas avant 1951. En attendant, la seule mesure effective est un fonds de secours qui finance des réalisations, à laquelle s'ajoute la pratique continue des commandes publiques. Voir Raymonde Moulin, L'artiste, l'institution et le marché [1992], P. : Flammarion, 1997, p. 145-155. 